\title{
Sarcoma-Like Pyogenic Granuloma of the Thumb and Respiratory Restrictive Syndrome in a Non-Compliant Hemodialysis Patient
}

\author{
Isabelle Simon $^{a} \quad$ Pierre Alain Gevenois $^{b}$ \\ Véronique Del Marmol ${ }^{c}$ Wissam El Kazzi $^{d}$ \\ Anne-Laure Trepant ${ }^{\mathrm{e}}$ Karine Gastaldello ${ }^{\mathrm{a}}$ \\ Joëlle L. Nortier ${ }^{a}$ \\ Departments of ${ }^{a}$ Nephrology, ${ }^{b}$ Radiology, ${ }^{c}$ Dermatology, ${ }^{d}$ Orthopedic Surgery, \\ and ${ }^{\mathrm{e}}$ Pathology, Erasme Hospital, Université Libre de Bruxelles, \\ Brussels, Belgium
}

\section{Key Words}

Brown tumor · Hemodialysis · Pyogenic granuloma - Secondary hyperparathyroidism

\begin{abstract}
Pyogenic granuloma is a benign vascular tumor of the skin or mucosae usually observed after irritative processes. We report the case of a non-compliant hemodialysis patient with severe hyperparathyroidism who rapidly developed growing pyogenic granuloma of the distal part of the left thumb. This tumor mimicked sarcoma and caused recurrent bleeding during hemodialysis sessions. Hand radiograph revealed an osteolytic lesion compatible with a brown tumor. Among other brown tumors, several of those found in the ribs were responsible of a severe respiratory restrictive deficit. This report highlights the difficulty to choose the adequate treatment of severe hyperparathyroidism, and discusses the benefit/risk balance of performing parathyroidectomy.
\end{abstract}

\section{Introduction}

Pyogenic granuloma is a benign tumor of the skin and mucosae of undetermined origin. Predisposing factors include trauma, hormonal disturbances, growth factors, infections, and microscopic arteriovenous anastomoses [1]. 
It may be hypothesized that destruction of bones located in subcutaneous region, such as observed in hyperparathyroidism (HPT), may lead to irritation of the skin and may favor the development of pyogenic granuloma.

Secondary HPT is one of the most common metabolic bone disorders in severe chronic kidney disease, particularly in end-stage renal disease (ESRD). HPT is due to multiple factors, including phosphate retention, altered metabolism of vitamin D, skeletal resistance to parathyroid hormone (PTH) and resistance to normal feedback mechanisms of PTH by calcium [2]. Histologically, resorption of cortical and trabecular bone is initially observed and is then followed by fibrosis. At an ultimate stage, brown tumors also named osteoclastomas may develop. Histological examination of the brown tumor found osteoclasts evolving in multinucleated giant cells and dispersed throughout fibrous tissue within the marrow space. Microfractures and microhemorrhages are also observed with hemosiderin-filled macrophages giving a brownish appearance $[2,3]$. These tumors represent a relatively rare $(1.5-1.7 \%)$ but potentially severe complication of secondary HPT, notably because of the risk of bone fractures and compression of contiguous structures [4]. They are usually seen in severe forms of HPT [4] and are observed more frequently in the long bones [5, 6], ribs [7-9] and pelvis but may occur in any bone such as jaw [4, 10-12], or vertebra $[2,13]$.

We report the case of a non-compliant woman on maintenance hemodialysis (HD) for about 10 years and presenting with multiple brown tumors related to severe HPT. A tumor on the left thumb has probably favored the occurrence of bleeding pyogenic granuloma and those of the ribs resulted in a severe pulmonary respiratory deficit. We discuss the medical as well as the surgical treatment difficulties of this case.

\section{Case Report}

Our patient was suffering from chronic kidney disease of unknown origin since 1998 (at the age of 47 years). She has always denied any medical management even for high arterial blood pressure. In October 2002, she developed ESRD necessitating maintenance HD. Serum level of intact PTH (iPTH) was increased at $465 \mathrm{pg} / \mathrm{ml}$, e.g. 6 folds the upper limit of the normal lab range $(8-74 \mathrm{pg} / \mathrm{ml}$, radioimmunoassay) associated with hypocalcemia and hyperphosphatemia. Calcium carbonate and calcitriol were prescribed. However, the patient's compliance to these drugs was almost absent during the following years. Phosphate binder (sevelamer hydrochloride) was also started in April 2003. Despite HD sessions of $12 \mathrm{~h}$ per week and prescription of cinacalcet in November 2008, serum iPTH and alkaline phosphatase (ALP) levels were not controlled.

In May 2010, the dermatologist diagnosed a sarcoma-like tumor on the left thumb (fig. 1a). This tumor grew very rapidly and caused recurrent bleeding during the HD sessions, despite minimal heparin doses used to prevent coagulation within the circuit. Skin biopsy performed in July 2010 showed vascular proliferation with infiltration by neutrophil polymorphonuclear cells. These histological findings are characteristic of pyogenic granuloma (fig. 1b). At this time, iPTH and ALP levels were $530 \mathrm{pg} / \mathrm{ml}$ (normal values: $17-73 \mathrm{pg} / \mathrm{ml}$, chemiluminescence assay) and 322 IU/l (normal values: 42-98 IU/l), respectively (fig. 1c). Radiograph of the left hand performed 1 year before had shown an osteolytic lesion of the second phalange of the thumb (fig. 1d). However, control radiograph in May 2010 (fig. 1e) showed the destruction of the second phalange. Because of the strong suspicion of underlying osteosarcoma, we finally convinced the patient to accept the surgical resection of this tumor 5 months later. Histological analysis of the surgical material $(5.9 \times 3.9 \times 3.7 \mathrm{~cm})$ showed giant multinucleated cells and ulceration partially filled by granulation tissue which is mainly composed of fibroblasts (fig. 1f). The postoperative course was uncomplicated.

Retrospective analysis of the medical file of this patient found out that she began to complain of knee pain in December 2009. Conventional radiographs revealed signs of tendon insertion disease but 
also the presence of a typical brown tumor $5 \mathrm{~cm}$ in diameter in the proximal shaft of the right tibia. The patient also developed severe dyspnea unrelated to fluid overload in May 2010. Respiratory functional tests clearly demonstrated a severe restrictive respiratory deficit [forced expiratory volume in one second $\left(\mathrm{FEV}_{1}\right)$ reduced to $23 \%$ of the predicted normal value, while the $\mathrm{FEV}_{1} /$ vital capacity ratio was normal]. Total lung capacity and functional residual capacity corresponded to 50 and $63 \%$ of predicted normal values, respectively. The chest expansion was measured during maximal inspiration and the patient was compliant. Plain radiograph of the chest showed a voluminous mass in the left hemithorax (fig. 2a). A two-dimensional computed tomography scan of the chest revealed the presence of ten costal lesions (five in both sides of the thoracic cage) corresponding to brown tumors. The most voluminous lesion was located on the posterolateral arch of the 7th left rib and measured 6 $\times 3.4 \mathrm{~cm}$ in horizontal diameter (fig. $2 \mathrm{~b}$ ).

Considering the comorbidities and the non-compliance of our patient, surgical removal of the parathyroid glands was not performed because of the major risk of postoperative hypocalcemia and the uncertain regression of the multiple brown tumors.

Since about 1 year, the patient is more compliant to treatment. Under medications including cinacalcet $90 \mathrm{mg} /$ day, alfacalcidol ( 3 pulses of $2 \mu \mathrm{g} / \mathrm{week}$ ), calcium carbonate $2 \mathrm{~g} /$ day, and sevelamer hydrochloride $2,400 \mathrm{mg} /$ day, HPT is better controlled: bioactive iPTH levels are between 120 and 550 $\mathrm{pg} / \mathrm{ml}$ (normal value $<37 \mathrm{pg} / \mathrm{ml}$, measured by chemiluminescence assay). The patient experiences less dyspnea. However, control respiratory functional tests performed in April 2012 showed the persistence of a restrictive respiratory deficit $\left(\mathrm{FEV}_{1}: 31 \%\right.$ of the predicted normal value and normal $\mathrm{FEV}_{1}$ /vital capacity ratio). A recently performed (August 2012) control two-dimensional computed tomography scan of the chest showed the disappearance of all non-calcified tumors. The calcified brown tumors had not progressed.

\section{Discussion}

The clinical presentation of these multiple brown tumors is very unusual in several aspects. First, the rapid growth of the pyogenic granuloma of the thumb was impressive as well as the recurrent bleedings during HD sessions despite minimized anticoagulation. Second, the development of a restrictive pulmonary deficit in association with multiple costal brown tumors is the first to be reported. These severe complications of secondary HPT are to be related to prolonged chronic uremic syndrome, late initiation of maintenance HD, and major non-compliance. The time course of the bone metabolism parameters recorded for several years is highly suggestive of these precipitating factors. In such cases, the location of brown tumors in limb extremities may result in skin barrier destruction and precipitate the occurrence of pyogenic granuloma.

Despite available medications such as cinacalcet, secondary HPT may remain difficult to treat and parathyroidectomy is still necessary in some cases [14]. A complete disappearance of the brown tumors has been observed after parathyroidectomy $[4,15]$. However, regression of the brown tumors can be incomplete $[3,10]$. Even if HPT is known to be the most important cause of brown tumors in ESRD, the lack of improvement in some patients after surgery suggests the contribution of other unknown factors in the development and growth of brown tumors [11]. The presence of supernumerary glands or residual clusters of parathyroid cells after surgery could be a possible explanation. The surgical removal of the brown tumor may be mandatory to prevent local, aggressive destruction of vital structures such as the jaw [11] and the spinal cord because of neurological compression [2]. Kidney transplantation and pulses of calcitriol have been shown to be successful in a 22-year-old patient with brown tumor of the rib [9]. In our patient, we remain skeptical 
regarding the absolute necessity of performing parathyroidectomy. In view of the risk of postoperative hypocalcemia, parathyroidectomy should be considered, not only for complications due to brown tumors, but also due to individual comorbidities and patient's compliance.

\section{Acknowledgements}

The authors thank Dr. Gervy Christine (Department of Medical Chemistry) who has taken care of the serum iPTH assays.
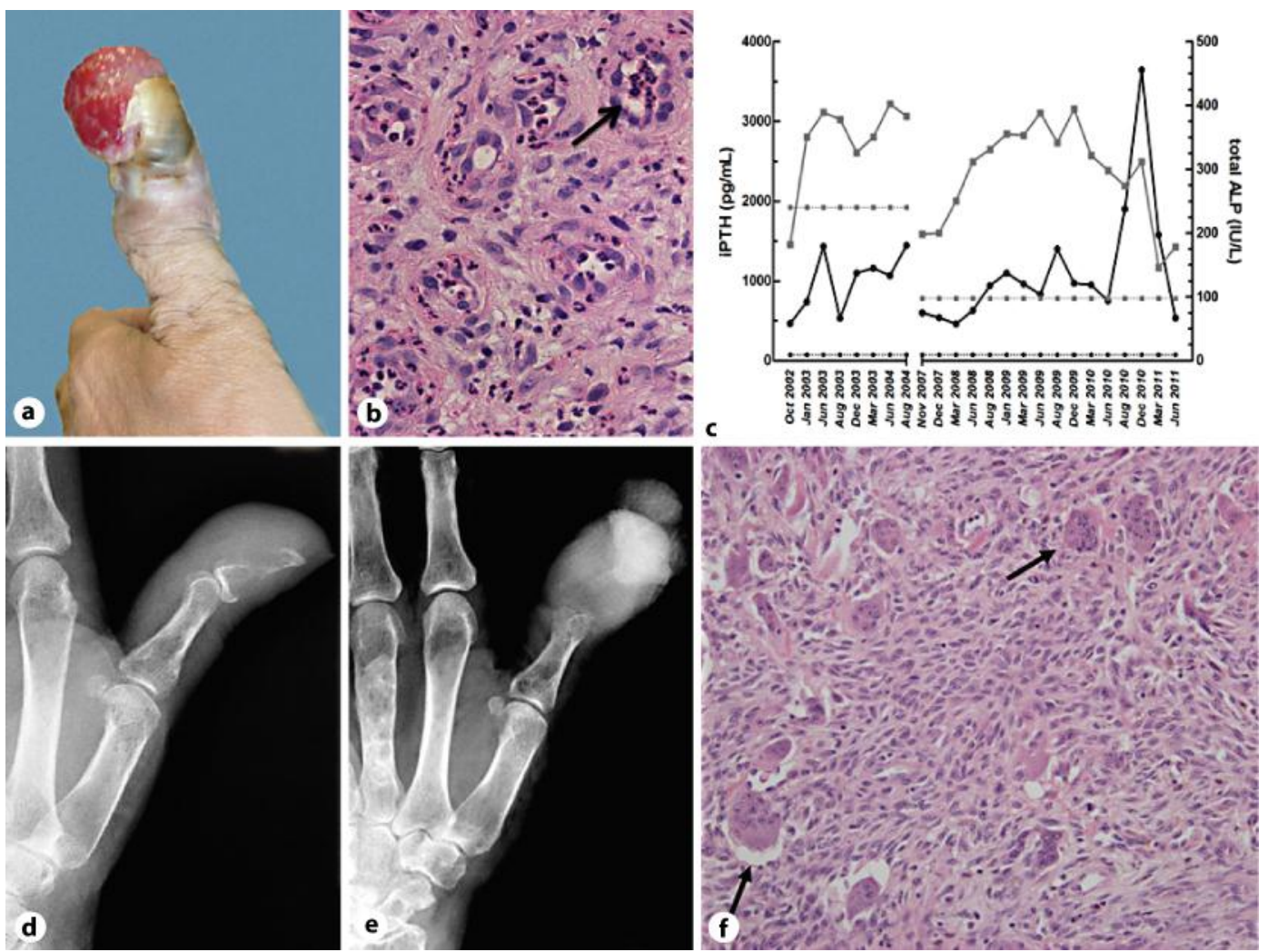

Fig. 1. a Macroscopic aspect of a sarcoma-like pyogenic granuloma of the left thumb. b Skin biopsy showing vessel proliferation with neutrophil polymorphonuclear cell infiltration (HE staining, $\times 400$ ). c Time course of serum iPTH and ALP levels. The dotted lines show upper normal values of iPTH $(-\bullet-)$ and ALP (-ם-). d Radiograph of the left hand (May 2009) showing an osteolytic lesion of the second phalange. e Control radiograph of the left hand (May 2010) showing voluminous tumefaction of soft tissues and bone destruction at the second phalange. $f$ Histological analysis of the resection material showing giant multinucleated cells (HE staining, $\times 200$ ). 

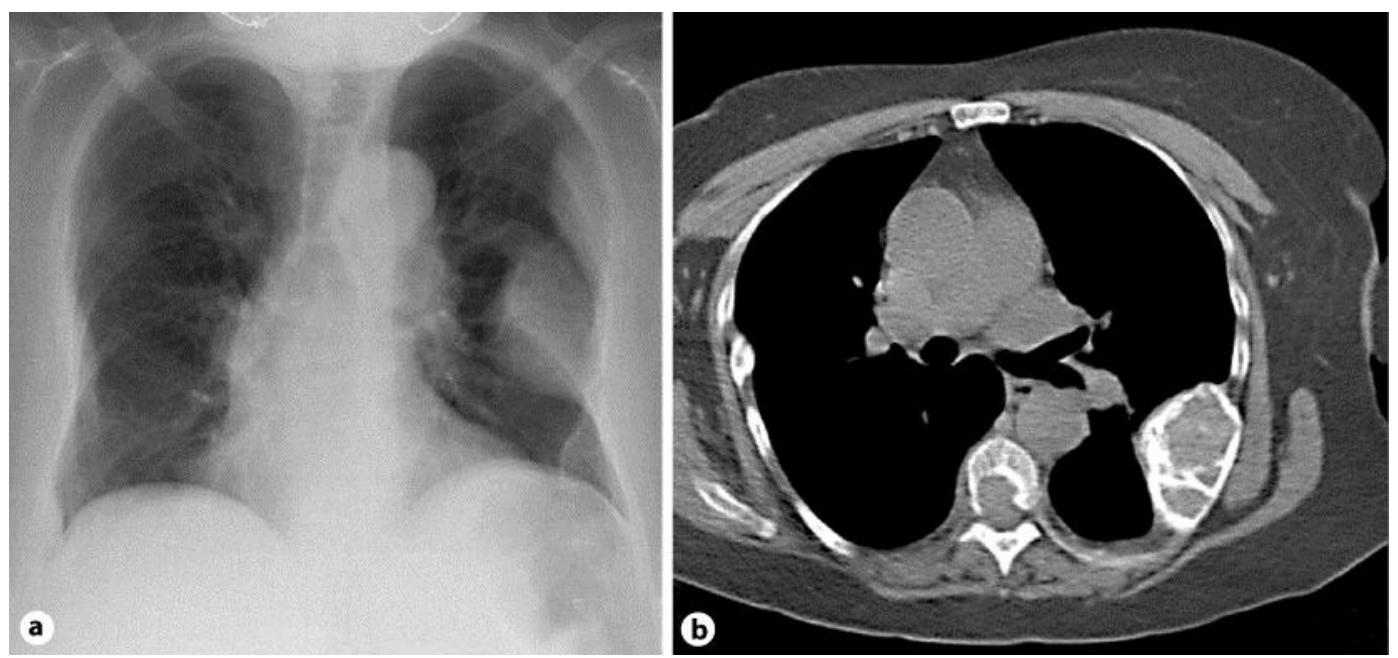

Fig. 2. a Plain radiograph of the chest showing a voluminous mass in the left side of the thoracic cage. b Two-dimensional computed tomography scan of the chest showing multiple bilateral costal brown tumors.

\section{References}

1 Ting PT, Barankin B: Smooth, soft, red papule on the lower lip. Can Fam Physician 2006;52:35-36.

2 Ren W, Wang X, Zhu B, Liu Z: Progressive paraplegia in a long-term haemodialysis patient. Am J Kidney Dis 2008;52:A37-A39.

3 Resic H, Masnic F, Kukavica N, Spasovski G: Unusual clinical presentation of brown tumor in hemodialysis patients: two case reports. Int Urol Nephrol 2011;4:575-580.

4 Di Daniele N, Condò S, Ferrannini M, Bertoli M, Rovella V, Di Renzo L, De Lorenzo A: Brown tumour in a patient with secondary hyperparathyroidism resistant to medical therapy: case report on successful treatment after subtotal parathyroidectomy. Int J Endocrinol DOI: 10.1155/2009/827652 (accessed May 19, 2011).

5 Yalcin MB, Hiz M, Can Unlu M, Dervisoglu S, Kanberoglu K, Bilge I, Ercan O: A case of brown tumor mimicking fibrous dysplasia in a patient with chronic renal failure. Acta Orthop Traumatol Turc 2008;42:296-301.

-6 Hong WS, Sung MS, Chun KA, Kim JY, Park SW, Lee KH, Lim HW, Lim YS, Yoo WJ, Chung MH: Emphasis on the MR imaging findings of brown tumor: a report of five cases. Skeletal Radiol 2011;40:205-213.

>7 Dursun H, Küçükosmanoglu O, Noyan A, Özbarlas N, Büyükçelik M, Soran M, Bayazit AK, Anarat A: Mitral annular calcification and brown tumor of the rib in a child with chronic renal failure. Pediatr Nephrol 2005;20:673-675.

8 Campuzano-Zuluaga G, Velasco-Pérez W, Marin-Zuluaga JI: A 60-year-old man with chronic renal failure and a costal mass: a case report and review of the literature. J Med Case Reports DOI: $10.4076 / 1752-$ 1947-3-7285 (accessed May 19, 2011).

-9 Spasovski G, Masin-Spasovska J, Gjurchinov D: Successful treatment of severe secondary hyperparathyroidism (brown tumor) by kidney transplantation and pulses of oral calcitriol. Clin Transplant 2009;23:426-430.

10 Benjelloun M, Tarrass F, Alaoui L, Medkouri G, Hachim K, Benghanem Garbi M, Ramdani B: Marked facial enlargement in secondary hyperparathyroidism. Nephrol Dial Transplant 2007;22:3082-3083.

-11 Tarrass F, Benjelloun M, Bensaha T: Severe jaw enlargement associated with uremic hyperparathyroidism. Hemodial Int 2008;12:316-318.

12 Pinto MC, Sass SM, Sampaio CP, Campos DS: Brown tumor in a patient with hyperparathyroidism secondary to chronic renal failure. Braz J Otorhinolaryngol 2010;76:404. 
13 Lacativa PG, Malzac Franco F, Pimentel JR, de Mattos Patricio Filho PJ, da Cruz Gonçalves MD, Farias ML: Prevalence of radiological findings among cases of severe secondary hyperparathyroidism. Sao Paulo Med J 2009;127:71-77.

14 Pitt SC, Sippel RS, Chen H: Secondary and tertiary hyperparathyroidism, state of the art surgical management. Surg Clin North Am 2009;89:1227-1239.

15 Nabi Z, Algailani M, Abdeslam M, Asaad L, Albaqumi M: Regression of brown tumor of the maxilla in a patient with secondary hyperparathyroidism after parathyroidectomy. Hemodial Int 2010;14:247-249. 